\title{
Biomechanics in Badminton- A Review
}

\author{
Manan Vora ${ }^{1,2 *}$, Manit Arora ${ }^{1,2}$ and Digpal Ranawat ${ }^{1}$ \\ ${ }^{1}$ Abhinav Bindra Targeting Performance, Mohali, India \\ ${ }^{2}$ Fortis Hospital, Mohali, India
}

Received: 制 October 08, 2018 Published: 制 October 17, 2018

*Corresponding author: Manan Vora, Abhinav Bindra Targeting Performance, Mohali, India

Abstract

Biomechanics plays an important role in badminton and a few studies over the years have improved our understanding of the sport. The aim of the current review is to summarize our current understanding with relation to the four common strokes (forehand serve, power stroke, forehand and backhand overhead strokes) and general endurance and fitness in badminton.

\section{Introduction}

Badminton is a racquet sport for two or four people, with a temporal structure characterized by actions of short duration and high intensity [1]. It originated in India towards the end of the nineteenth century during British colonial rule and was played at military bases in multiple Indian cities [2]. It is recognised that a combination of excellence in technical, tactical, anthropometrical, physical and mental skills is needed for elite badminton performance [3].

The application of sport science to coaching has become the single most important factor behind the rapid advances made in international sport performances during the past 20 years [4]. Biomechanics is the physics of human motion, and the factors that cause this movement. It explains how sport technique may be analysed and the resulting information used to improve athletic performance [4]. More importantly, biomechanics explains the inter-relationships between the athlete's structure, physical capacity and his/her unique technique [4]. Incorporating the latest principles and practices of biomechanics will assist high-level coaches and sport scientists prepare their athletes for competition [4]. Biomechanics in badminton has been studied with relation to power strokes, forehand overhead jump smash, backhand overhead strokes and forehand serves.

\section{Power Strokes}

With respect to badminton power strokes, only hypothetical evidence was available until in-depth biomechanical studies on badminton performance were carried out. It was earlier believed that badminton strokes were made with a wrist snap [5]. showed that all badminton strokes are made with forearm rotation rather than wrist snap [5]. Further work showed the importance of radio- ulnar pronation, elbow extension, and wrist ulnar deviation [6]. An estimation of joint contributions made to the velocity of the shuttlecock in the badminton smash attributed $53 \%$ of the final output to shoulder rotation and radio-ulnar pronation [7]. Hence, the wrist snap belief was soon abolished. In a review study on underarm power strokes, subsequent qualitative analysis revealed that world class players gain a major proportion of power for the underarm forehand and clear high serve by pronating the forearm and medially rotating the upper arm [8]. The authors also found that backhand clears are performed using supination of the forearm and lateral rotation of the upper arm. The same authors have conducted various studies and presented multiple research papers that expanded on the concepts related to badminton stroke production. Thus, there is a trend away from assuming a superior role of the wrist in power strokes to an inferior role. It appears that the elbow and shoulder contribute the majority of power in a power stroke. It is yet to be determined the contribution of individual muscle groups to the power component and this maybe an avenue of future research with the potential of activating certain muscle groups to improve performance.

\section{Forehand Overhead Jump Smash}

Studies have been conducted on the biomechanics of the forehand overhead jump smash, a lethal weapon used by every badminton player. Smash and jump smash performances were studied and it was noted that faster shuttle velocity of the jump smash may result from greater elbow angular velocity [9]. One study aimed to establish the temporal-spatial relationship between muscle activity and the smash stroke of skilled badminton players and concluded that controlling the distal muscles of the upper limb appears to be important for achieving accurate performance of the 
smash in badminton [10]. A study conducted on the biomechanics of the forehand and backhand stroke showed that skilled players reached significantly higher angular velocities for glenohumeral external rotation, elbow supination and wrist extension in the backhand stroke, compared to the less skilled players, but there were no such differences in the forehand stroke [11].This suggests that although forehand is the more important stroke, the backhand is the one that players are working on as their career progresses. One study analysed the surface EMG activity of upper extremities between smash and jump smash by elite badminton players using two digital video cameras to obtain the 3D kinematics data of shuttlecock and measured the surface EMG signals of seven upper limb muscles [12]. The authors reported that there was no significant difference between the two smashes in initial shuttle velocity, but there was significant difference between the two smashes in the sequence of the surface EMG activity of the upper limb and that the jump smash exerted higher EMG activity than smash in the phase before contact point. Thus, it appears that preimpact EMG activation is the most important part of shuttle velocity, which has major implications for performance. It will be beneficial to assess whether improving pre-smash activation improves shuttle velocity speeds of smash.

\section{Backhand Overhead Strokes}

An analysis of three different backhand overhead strokes (smash, clear and drop) focused on the phase of preparation position to the point of making contact with the shuttle [13]. The authors found that increasing the shoulder angle of drop and the wrist angular velocity of smash and clear will increase the shuttle velocity.

\section{Forehand Serves}

A study on the analysis of arm movement in badminton forehand long and short service showed that there were significant differences between long and short service in elbow angle, shuttle height at contact and shuttle attaining maximum height [14]. Thus, the position of serve determines maximum height of serve which appears to be mediated by shuttle height and elbow angle at the time of service.

\section{General Endurance and Fitness}

The need of muscular endurance combined with appropriate maximal and explosive muscle strength in elite badminton players is gaining importance [15]. The authors determined maximal muscle strength of the knee extensor (quadriceps) and flexor muscles (hamstrings) using isokinetic dynamometry and explosive muscle strength was measured by determining the contractile rate of force development during maximal isometric muscle contractions. They showed that musculature of the lower extremities is especially important since rapid and forceful movements with the weight of the body are performed repeatedly throughout a match. A further study found that the intermittent actions during a game are demanding on the aerobic (60-70\%) as well as anaerobic systems $(30 \%)$ with greater demand on the alactic metabolism with respect to the lactic anaerobic metabolism [1].

\section{References}

1. Phomsoupha M, Laffaye G (2015) The science of badminton: Game characteristics, anthropometry, physiology, visual fitness and biomechanics. Sports Medicine 45(4): 473-495.

2. Guillain, Jean Yves (2018) Badminton: An Illustrated History. Jean-Yves Guillain Public book Anglais Broche.

3. Elferink Gemser M, Jordet G, Coelho e Silva M, Visscher C (2011) The marvels of elite sports: How to get there? British Journal of Sports Medicine 45: 683-684.

4. Ackland TR, Elliott B, Bloomfield J (2009) Applied Anatomy and Biomechanics in Sport. Human Kinetics.

5. Poole JR (1970) A Cinematographic Analysis of the Upper Extremity Movements of World Class Players Executing Two Basic Badminton Strokes pp. 100.

6. Sakurai S, Ikegami Y, Yabe K (2008) A Three-Dimensional Cinematographic Analysis of Badminton Strokes. ISBS-Conference Proceedings Archive 1(1)

7. Waddell DB, Gowitzke BA (2000) Biomechanical Principles Applied to Badminton Power Strokes. ISBS - Conference Proceedings Archive 1(1).

8. Gowitzke BA, Waddell DB (1989) Biomechanical studies of badminton underarm. Power strokes, court movement, and flexibility: A review. ISBS - Conference Proceedings.

9. Tsai CL, Chang SS, Huang C (1998) Biomechanical Analysis of Differences in Badminton Smash and Jump Smash and Jump Smash. ISBS- Conference Proceedings Archive 1(1)

10. Sakurai S, Ohtsuki T (2000) Muscle activity and accuracy of performance of the smash stroke in badminton with reference to skill and practice. Journal of Sports Sciences 18(11): 901-914.

11. Sørensen K (2010) A Biomechanical Analysis of Clear Strokes in Badminton Executed by Youth Players of Different Skill Levels. Master Thesis, Sports Science Aalborg University.

12. Tsai CL, Yang, CC, Lin MS, Huang KS (2008) The Surface Emg Activity Analysis Between Badminton Smash and Jump Smash. ISBS - Conference Proceedings Archive.

13. Huang KS, Huang C, Chang SS, Tsai CL (2002) Kinematic Analysis of Three Different Badminton Backhand Overhead Strokes p. 3.

14. Ahmed S, Bari MA, Ahmad A, Mohammad A, Khan A et al. (2011) Analysis of Arm Movement in Badminton of Forehand Long and Short Service. Innovative Systems Design and Engineering 2(3): 7.

15. Andersen L, Larsson B, Overgaard H, Aagaard P (2007) Torque Velocity characteristics and contractile rate of force development in elite badminton players. European Journal of Sport Science - EUR J SPORT SCI 7(3): 127-134. 
This work is licensed under Creative Commons Attribution 4.0 License

To Submit Your Article Click Here:

Submit Article

DOI: $10.32474 /$ OSMOAJ.2018.02.000129

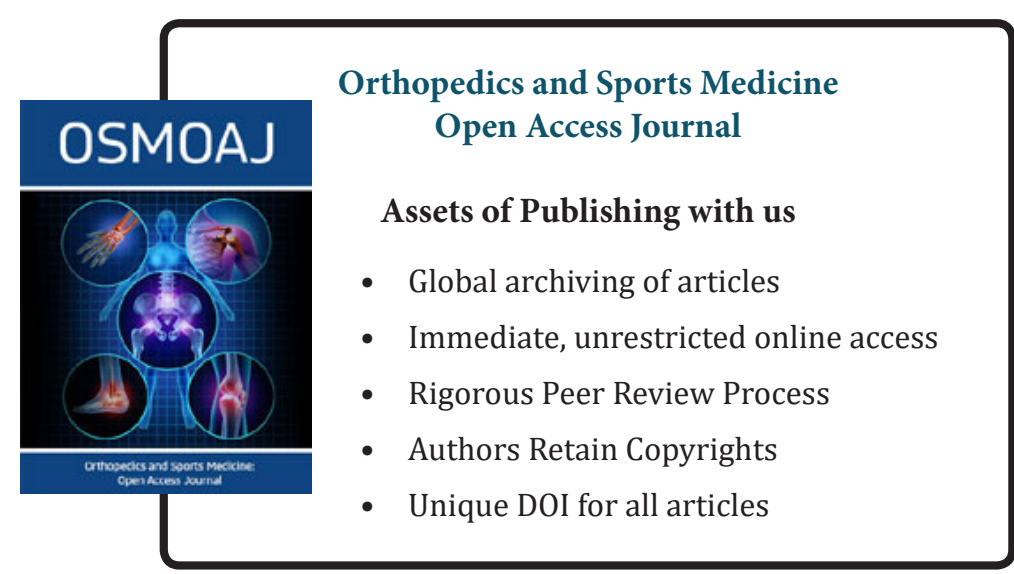

